\title{
PRO-NILPOTENT REPRESENTATION OF HOMOLOGY TYPES
}

\author{
EMMANUEL DROR
}

\begin{abstract}
The completion tower $R_{n} X$ defined by Bousfield and Kan is shown to preserve the homology with $R$-coefficients. This property of preserving $R$-homology characterizes the tower completely [4].
\end{abstract}

0 . Introduction. For a given space $X$ and a solid ring $R$ (i.e., a commutative ring with 1 for which the multiplication map $R \otimes R \rightarrow R$ is an isomorphism), Bousfield and Kan define $R_{\infty} X$-the $R$-completion of $X$. The functor $R_{\infty} X=$ proj lim $R_{n} X$ [3] has very nice properties and generalizes all the other partial and nonfunctorial construction of completion and localizations.

One of the most important unsolved problems with regard to $R_{\infty}$ is the relationship between $H_{*}(X, R)$ and $H_{*}\left(R_{\infty} X, R\right)$. It is known [2] that if $X$ is an infinite wedge of circles then the natural map $H_{*}(X, Z) \rightarrow H_{*}\left(Z_{\infty} X, Z\right)$ is not an isomorphism. But nothing is known if $X$ is, e.g., a wedge of two circles.

The purpose of this note is to show that one always gets a "prohomology isomorphism" if one substitutes for $R_{\infty} X$ the whole tower $\left(R_{n} X\right)_{n}$. In fact, we will prove that for any fixed $k \geqq 0$ the map $H_{k}(X, R) \rightarrow$ $\left(H_{k}\left(R_{n} X, R\right)\right)_{n}$, where the target is a tower of abelian groups-considered as a pro-group, is an isomorphism of pro-groups. In particular,

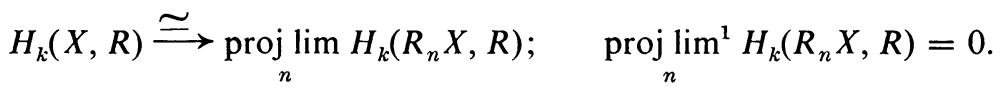

This is equivalent to

inj $\lim H^{k}\left(R_{n} X, M\right)=H^{k}(X, M)$ for any $R$ module $M$.

1. Pro-groups, pro-homotopy type. We will consider the category whose objects are towers:

$$
\left(K_{n}\right)_{n}=\cdots \rightarrow K_{i} \rightarrow K_{i-1} \rightarrow \cdots \rightarrow K_{0}
$$

Received by the editors May 30, 1972.

AMS (MOS) subject classifications (1970). Primary 55D15.

(c) American Mathematical Society 1973 
of simplicial sets with one vertex or of groups. The morphisms are given by

$$
\operatorname{Hom}\left(\left(K_{n}\right)_{n},\left(L_{m}\right)_{m}\right)=\underset{m}{\operatorname{proj}} \lim \operatorname{inj} \lim _{n} \operatorname{Hom}\left(K_{n}, L_{m}\right)
$$

and can be thought of as a commutative ladder $\left(K_{n}\right)_{n} \rightarrow\left(L_{m}\right)_{m}$.

A short introduction to the category of pro-objects is given in the Appendix of [1], in which a theory of pro-homotopy type is developed. Another approach to the homotopy type of diagram is given in [4]. A more extensive treatment of pro-objects over an abelian category is given in [6], [4].

The pro-nilpotent completion of a tower $\left(X_{n}\right)_{n}$ of "connected spaces", i.e., of simplicial sets with one vertex, is given by the tower $R_{n} X_{n}$ where the map $R_{n} X_{n} \rightarrow R_{n-1} X_{n-1}$ is the composition $R_{n} X_{n} \rightarrow R_{n} X_{n-1} \rightarrow R_{n-1} X_{n-1}$. Thus for every such pro-simplicial set $X_{n}$ one has a natural map of towers:

$$
\left(X_{n}\right) \rightarrow\left(R_{n} X_{n}\right) \text {. }
$$

2. THEOREM. Let $\left(X_{n}\right)_{n}$ be a tower of "connected spaces"; then for every $k \geqq 0$ the map $\left(H_{k}\left(X_{n}, R\right)\right)_{n} \rightarrow\left(H_{k}\left(R_{n} X_{n}, R\right)\right)_{n}$ is an isomorphism in the category of pro-groups.

2.1. Lemma. The map $\left(X_{n}\right)_{n} \rightarrow\left(R_{n} X_{n}\right)_{n}$ induces an isomorphism of $R$ homology if and only if the map $\left(R_{n} X_{n}\right)_{n} \rightarrow\left(R_{n} R_{n} X_{n}\right)_{n}$ is a homotopy equivalence of pro-simplicial sets.

Proof. The argument is the same as in [4]: One looks at the square of abelian pro-groups

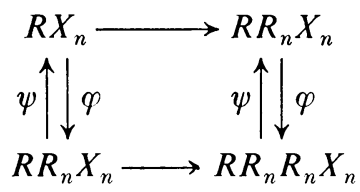

which exists and commutes by virtue of the triple structure of the functor $R_{s}$.

Since the bottom map is an equivalence and $\psi \varphi=\mathrm{id}$, it follows that the top map is an equivalence also, i.e., $H_{k}\left(X_{n}, R\right) \simeq \pi_{k} R X_{n} \rightarrow H_{k}\left(R_{n} X_{n}, R\right)$ is an isomorphism of pro-groups.

2.2. Proof of the Theorem. According to Lemma 2.1 it suffices to prove that the map $\left(R_{n} X_{n}\right)_{n} \rightarrow\left(R_{n} R_{n} X_{n}\right)_{n}$ is a homotopy equivalence of towers. Since the tower of the target is cofinal [1] in the pro-simplicial set $\left(R_{s} R_{n} X_{n}\right)_{s, n}$, it is enough to show that for any fixed $n$ the map

$$
R_{n} X_{n} \stackrel{\sim}{\longrightarrow}\left(R_{s} R_{n} X_{n}\right)_{s} \quad(n \text {-fixed })
$$

is a homotopy equivalence of towers, when we regard $R_{n} X_{n}$ as a constant tower. 
We prove $(*)$ by showing that for every fixed $n, k \geqq 0$, the map $\pi_{k} R_{n} X \rightarrow\left(\pi_{k} R_{s} R_{n} X_{n}\right)_{s}$ is an isomorphism of pro-groups.

One first notes that, as A. K. Bousfield observed, it follows from the convergence of the homotopy spectral sequence [4] that for any $k, s \geqq 0$ there exists $N=N(s, Y, k)$ such that

$$
\text { Image }\left(\pi_{k} R_{\infty} Y \rightarrow \pi_{k} R_{s} Y\right)=\operatorname{Image}\left(\pi_{k} R_{n} Y \rightarrow \pi_{k} R_{s} Y\right) .
$$

If one puts $Y=R_{n} X$ which is $R$-nilpotent, one gets $Y \stackrel{\cong}{\rightarrow} R_{\infty} Y$. Thus it follows from the diagram

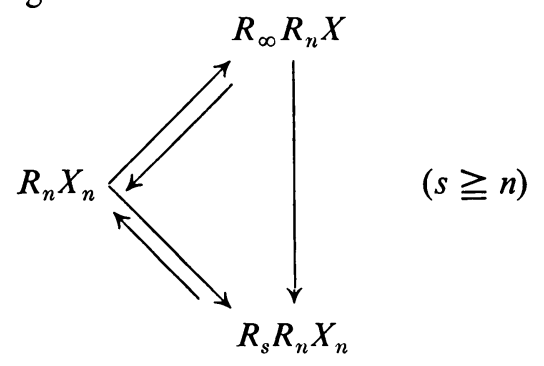

that for $s \geqq n$,

$$
\operatorname{Im}\left(\pi_{*} R_{\infty} R_{n} X_{n} \rightarrow \pi_{*} R_{s} R_{n} X_{n}\right) \simeq \pi_{*} R_{n} X_{n} .
$$

Thus, for some $N$,

$$
\operatorname{Im}\left(\pi_{*} R_{n} R_{n} X_{n} \rightarrow \pi_{*} R_{s} R_{n} X\right) \simeq \pi_{*} R_{n} X .
$$

Now for $k>1$, it follows from the triple structure of $R_{n}$ that for each $s \geqq n$ one has a natural decomposition

$$
\pi_{k} R_{s} R_{n} X_{n} \simeq \pi_{k} R_{n} X_{n} \oplus J_{s}
$$

Thus one gets from the above that for any $s \geqq n$ there exists $N \geqq s$ such that the map $J_{X} \rightarrow J_{s}$ is the trivial map, which means that the pro-object $\left(\pi_{k} R_{s} R_{n} X\right)_{s} \simeq\left(\pi_{k} R_{n} X \oplus J_{s}\right)_{s}$ is isomorphic to the constant pro-object $\pi_{k} R_{n} X$ (see $\S 5$ of [6]).

As for the case $k=1$, one uses the same argument for the quotient $\Gamma_{r} / \Gamma_{r+1} \pi_{1}$ of the lower central series and the fact that $\pi_{1}$ is nilpotent, i.e., that series is finite, to arrive at the same conclusion. This completes the proof.

3. Corollaries. We will now restrict ourself to the case when $\left(X_{n}\right)_{n}$ is a constant tower, i.e., $X_{n}=X$.

3.1. Corollary. For every connected space $X$ and $k \geqq 0, H_{k} X \rightarrow$ $\left(H_{k} R_{n} X\right)_{n}$ is an isomorphism of pro-groups.

Thus in particular one has the isomorphisms of groups:

$$
H_{k} X \stackrel{\cong}{\longrightarrow} \underset{n}{\operatorname{proj} \lim } H_{k} R_{n} X, \quad 0 \stackrel{\simeq}{\longrightarrow} \underset{n}{\operatorname{proj} \lim ^{1}} H_{k} R_{n} X .
$$


3.2. Pro-nilpotent completion à la Artin-Mazur. One can consider the functor which assigns to every map $X \rightarrow N$ wherc $N$ is an $R$-nilpotent space the space $N$, and to every commutative triangle

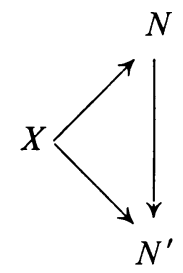

with $N, N^{\prime}$ nilpotent spaces the map $N \rightarrow N^{\prime}$. Thus one gets a pro-object $N_{R} X$ in the sense of [1], because the category of maps $X \rightarrow N$ with morphism the above triangles is a filtering category [1]: The product of two $R$ nilpotent spaces are nilpotent and so is the connected equalizer of two maps $N \rightrightarrows N^{\prime}[\mathbf{5}]$.

3.3. Proposition. For every connected space $X$, the tower $R_{n} X$ is cofinal in the pro-object $N_{R} X$.

Proof. Clearly $X \rightarrow N_{R} X$ is $R$-cohomology isomorphism since $k(R, n)$ are $R$-nilpotent. Since $X \rightarrow R_{n} X$ is $R$-homology isomorphism it is $R$ cohomology isomorphism. Since all the target spaces are $R$-nilpotent one gets a map $R_{n} X \rightarrow N_{R} X$ of pro-objects, which induces isomorphism on homotopy groups by the generalized Whitehead theorem [5] applied to towers of nilpotent spaces.

\section{REFERENCES}

1. M. Artin and B. Mazur, Etale homotopy, Lecture Notes in Math., no. 100, Springer-Verlag, Berlin and New York, 1969. MR 39 \#6883.

2. A. Bousfield and D. Kan, Localization and completion in homotopy theory, Bull. Amer. Math. Soc. 77 (1971), 1006-1010.

3. - - Homotopy with respect to a ring, Proc. Sympos. Pure Math., vol. 22, Amer. Math. Soc., Providence, R.I., 1971, pp. 59-64.

4. —_ Homotopy limits, completions and localizations, Lecture Notes in Math., Springer-Verlag, Berlin and New York, 1972.

5. E. Dror, A generalization of the Whitehead theorem, Sympos. on Algebraic Topology, P. Hilton (editor), Lecture Notes in Math., no. 249, Springer-Verlag, New York, 1971.

6. J. Duskin, Pro-objects, Seminar Heidelberg, Strasbourg, Inst. de Recherche Math. avancée, Strasbourg, 1966-67, Exposé 6.

Department of Mathematics, Graduate Center (CUny), New York, New YORK 10036 